\title{
Exploring and Reconstructing Ancestral Anatomies using Ontology-Informed Approaches
}

\author{
Sergei Tarasov ${ }^{\ddagger}$, , Istvan Mikol, Matthew Yoder", Josef Uyeda ${ }^{\S}$ \\ ‡ Finnish Museum of Natural History, Helsinki, Finland \\ $\S$ Virginia Tech, Blacksburg, United States of America \\ | University of New Hampshire, Durham, United States of America \\ Tा University of Illinois, Champaign, United States of America
}

Corresponding author: Sergei Tarasov (sergxf@yandex.ru)

Received: 10 Jun 2019 | Published: 13 Jun 2019

Citation: Tarasov S, Miko I, Yoder M, Uyeda J (2019) Exploring and Reconstructing Ancestral Anatomies using

Ontology-Informed Approaches. Biodiversity Information Science and Standards 3: e37038.

https://doi.org/10.3897/biss.3.37038

\begin{abstract}
Ancestral character state reconstruction has been long used to gain insight into the evolution of individual traits in organisms. However, organismal anatomies (= entire phenotypes) are not merely ensembles of individual traits, rather they are complex systems where traits interact with each other due to anatomical dependencies (when one trait depends on the presence of another trait) and developmental constraints. Comparative phylogenetics has been largely lacking a method for reconstructing the evolution of entire organismal anatomies or organismal body regions. Herein, we present a new approach named PARAMO (Phylogenetic Ancestral Reconstruction of Anatomy by Mapping Ontologies, Tarasov and Uyeda 2019) that takes into account anatomical dependencies and uses stochastic maps (i.e., phylogenetic trees with an instance of mapped evolutionary history of characters, Huelsenbeck et al. 2003) along with anatomy ontologies to reconstruct organismal anatomies.
\end{abstract}

Our approach treats the entire phenotype or its component body regions as single complex characters and allows exploring and comparing phenotypic evolution at different levels of anatomical hierarchy. These complex characters are constructed by ontology-informed amalgamation of elementary characters (i.e., those coded in character matrix) using stochastic maps. In our approach, characters are linked with the terms from an anatomy 
ontology, which allows viewing them not just as an ensemble of character state tokens but as entities that have their own biological meaning provided by the ontology. This ontologyinformed framework provides new opportunities for tracking phenotypic radiations and anatomical evolution of organisms, which we explore using a large dataset for the insect order Hymenoptera (sawflies, wasps, ants and bees).

\section{Keywords}

comparative phylogenetics, ontologies, organismal anatomies, trait evolution, Hymenoptera

\section{Presenting author}

Sergei Tarasov

\section{Presented at}

Biodiversity_Next 2019

\section{References}

- Huelsenbeck J, Nielsen R, Bollback J (2003) Stochastic Mapping of Morphological Characters. Systematic Biology 52 (2): 131-158. https://doi.org/10.1080/10635150309342 - Tarasov S, Uyeda J (2019) PARAMO: Phylogenetic Ancestral Reconstruction of Anatomy by Mapping Ontologies. GitHub. URL: https://github.com/sergeitarasov/PARAMO 